\title{
An Artificial Neutral Network (ANN) Model for Predicting Biodiesel Kinetic Viscosity as a Function of Temperature and Chemical Compositions
}

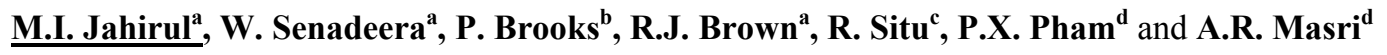 \\ ${ }^{a}$ Biofuel Engine Research Facility, Science and Engineering Faculty, Queensland University of Technology \\ (QUT), Brisbane, Australia \\ ${ }^{b}$ School of Science, Education and Engineering, University of the Sunshine Coast, Australia \\ ${ }^{c}$ School of Engineering and Physical Science, James Cook University, Townsville, Queensland \\ 4814, Australia \\ ${ }^{d}$ School of Aerospace, Mechanical and Mechatronic Engineering, University of Sydney NSW 2006 Australia \\ Email: md jahirul@yahoo.com ;mi.islam@student.qut.edu.au
}

\begin{abstract}
An Artificial Neural Network (ANN) is a computational modeling tool which has found extensive acceptance in many disciplines for modeling complex real world problems. An ANN can model problems through learning by example, rather than by fully understanding the detailed characteristics and physics of the system. In the present study, the accuracy and predictive power of an ANN was evaluated in predicting kinetic viscosity of biodiesels over a wide range of temperatures typically encountered in diesel engine operation. In this model, temperature and chemical composition of biodiesel were used as input variables. In order to obtain the necessary data for model development, the chemical composition and temperature dependent fuel properties of ten different types of biodiesels were measured experimentally using laboratory standard testing equipments following internationally recognized testing procedures. The Neural Networks Toolbox of MatLab R2012a software was used to train, validate and simulate the ANN model on a personal computer. The network architecture was optimised following a trial and error method to obtain the best prediction of the kinematic viscosity. The predictive performance of the model was determined by calculating the absolute fraction of variance $\left(R^{2}\right)$, root mean squared $(R M S)$ and maximum average error percentage (MAEP) between predicted and experimental results. This study found that ANN is highly accurate in predicting the viscosity of biodiesel and demonstrates the ability of the ANN model to find a meaningful relationship between biodiesel chemical composition and fuel properties at different temperature levels. Therefore the model developed in this study can be a useful tool in accurately predict biodiesel fuel properties instead of undertaking costly and time consuming experimental tests.
\end{abstract}

Keywords: $\quad$ ANN model, Biodiesel, Kinematic viscosity, Temperature dependence 
Jahirul et al., An Artificial Neutral Network (ANN) Model for Predicting Biodiesel Kinetic Viscosity as a Function of Temperature and Chemical Compositions

\section{INTRODUCTION}

Vegetable oil methyl or ethyl esters, commonly referred to as 'biodiesel', are a renewable liquid fuel alternative to petroleum diesel. They are made from agricultural products, forest organic matter and animal fat feedstocks. In technical terms, biodiesel is diesel engine fuel comprised of mono-alkyle esters of long chain fatty acids derived from vegetable oil or animal fats, designated B100 and meeting the requirements of ASTM D 6751or EN 14214 (Demirbas, 2008). These mono-alkyl esters are the main chemical species that give biodiesel similar or better fuel properties compared with petroleum diesel (Fernando et al., 2007).

Biodiesel is considered as an alternative transport fuel made from domestically produced oilseed crops and animal fat which can be directly used on conventional unmodified diesel engines. Biodiesel is safe to handle, store and transport because it is biodegradable, non-toxic and has a high flash point compared to that of petroleum diesel fuel. One of the major advantages of biodiesel is that it has potential to reduce dependency of imported petroleum. In fuel property terms biodiesel usually has a higher cetane rating compared to that of petroleum diesel which improves engine performance. In addition has a much better lubricant properties than petroleum diesel and which can extend engine life (Haseeb et. al., 2011). Biodiesel is more environmentally friendly due to its clean burning properties. It can reduce emission up to $75 \%$ than conventional diesel fuel, and substantially reduces unburned hydrocarbons, carbon monoxides and particulate matters, and also eliminates sulphur dioxide emissions in exhaust emissions. The exhaust emissions of particulate matter by mass from biodiesel have been found to be 30\% lower than overall particulate matter emissions from fossil diesel. The exhaust emissions of total hydrocarbons are up to $93 \%$ lower for biodiesel than diesel fuel (Hoekman et. al., 2012).However, as a fuel, there are currently several disadvantages to using biodiesel in diesel engine applications. The major disadvantage is its higher kinematic viscosity compared to conventional diesel fuel especially at low temperature. These differences mainly result from the difference in chemical compositions between petroleum diesel and biodiesel (Jahirul et. al., 2013). Viscosity is one of the most critical features of a liquid fuel which is defined as the resistance to shear or flow. It is also highly dependent on temperature and it describes the behavior of a liquid in motion near a solid boundary such as the walls of a pipe. It plays a dominant role in fuel atomization and therefore the fuel-air mixture quality, combustion, and emission characteristics. (Lee et. al., 2002; Knothe and Steidle, 2005). On the other hand, very low fuel viscosity is not desirable because the fuel then doesn't provide sufficient lubrication for the precision fit of fuel injection pumps, resulting in leakage or increased wear. Therefore, all biodiesel standards define the upper and lower limits for the kinematic viscosity of biodiesel.

The kinematic viscosity of biodiesel is dependent on its complex chemical composition. As the lengths of the fatty acid chain in the ester molecules increased, so did the degree of random intermolecular interactions which eventually increases kinematic viscosity of biodiesel. The effect becomes more evident at lower temperatures, where molecular movements are more restricted (Knothe and Steidle, 2007). Other factors that influence biodiesel viscosity include: number of double bonds, molecular weight, branching hydroxyl groups and the amount of impurities, such as unreacted glycerides or glycerol, amount of Carbon (C), Oxygen (O), Hydrogen (H) etc (Refaat, 2009).

In recent years, ANN modeling techniques have gained in popularity due to their ability to accurately predict from small data sets (examples) rather than from larger data sets requiring costly and time-consuming studies and experiments. ANN has been successfully applied in various disciplines, including neuroscience (Alkim et. al., 2012), mathematical and computational analysis (Costa et. al., 2012), learning systems (Carrillo et. al., 2012), combustion (Christo et. al., 1996) and engineering design and application (Minnett et. al., 2011). In the existing literatures, it was shown that the use of ANN is a powerful modeling tool that has the ability to identify complex relationships from input-output data. However, no investigation was found to develop ANN model to predict the kinematic viscosity of biodiesel at different temperature. Such an ANN prediction model has been developed based on the chemical composition of biodiesel. The developed ANN model has been simulated with new input data and prediction ability was presented graphically.

\section{ARTIFICIAL NEURAL NETWORKS (ANN)}

The foundation of artificial neural networks (ANN) in a scientific sense begins with a biological neuron. In the brain, there is a flow of coded information (using electrochemical media, the so-called neurotransmitters) from the synapses towards the axon. The axon of each neuron transmits information to a number of other 
neurons. Groups of neurons are organised into sub-systems and the integration of these sub-systems forms the brain. On the other hand, an ANN is composed of a large number of simple processing units called neurons which are fully connected to each other through adoptable synaptic weight. In the training process, weights are adjusted to minimise the error between actual output and desired output (Basheer and Hajmeer, 2000).

The most important feature of artificial neural networks is their ability to solve problems through learning by example, rather than by fully understanding the detailed characteristics of the systems. This feature makes it very useful because it works like a 'black box' model, whereby the ANN does not require detail or complete information about the problem, but can be formed with sets of data inputs and outputs. It has a natural propensity to store experiential knowledge and to make it available for use (Figure 1). Therefore, this nonlinear computer algorithm can model large and complex systems with many interrelated parameters (Arcakhoglu et. al., 2004).

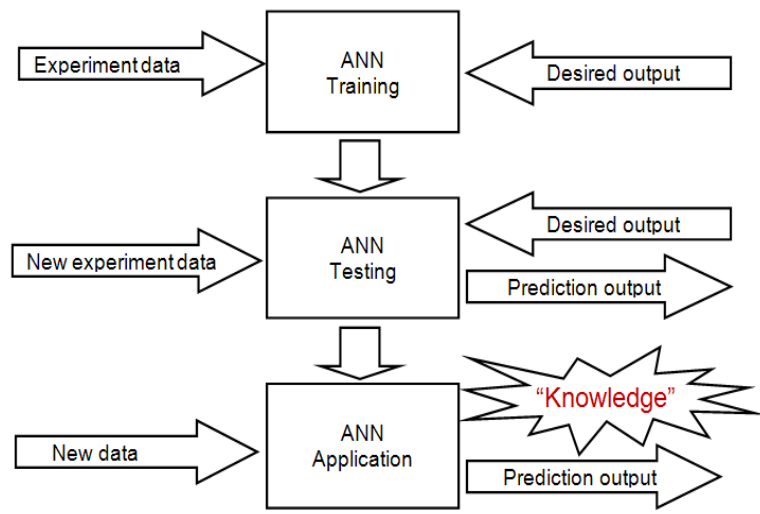

Figure 1. Working principle of ANN (Jahirul et. al., 201

\section{METHODOLOGY}

Biodiesel is made in a chemical process called transesterification, where organically derived oils or fat are combined with alcohol (usually methanol) and chemically altered to form fatty esters such as methyl ester. The chemical composition and fatty acid profile of biodiesel is identical to that of its parent oil or fat. The properties of biodiesel fuel are also determined by the amounts of individual fatty acid ester that are present in the molecules. In the present study, experiments were conducted on 10 biodiesel samples to develop ANN prediction model for kinematic viscosity in different temperature condition. Among the samples, biodiesel derived from canola oil (COME), cotton seed oil (CSOME) and Soybean oil (SOME) are commercially available. The other biodiesel samples such as POME-1, POME-2, POME-3, POME-4, POME-5, POME-6 and POME-7 are produced by fractionating of palm oil biodiesel.

Chemical composition and fatty acid profile of biodiesel samples were analysed using Gas chromatographymass spectrometry (GC-MS) following EN 14103 standards. In this process, biodiesel samples were diluted 1:100 with n-hexane. Sample injections were 1uL into a PerkinElmer Clarus 580 GC-MS fitted with Elite $5 \mathrm{MS}, 30 \mathrm{~m} \times 0.25 \mathrm{~mm} \times 0.25 \mathrm{um}$ column. The split ratio was $30: 1$ with a column flow of $1 \mathrm{~mL} / \mathrm{min} \mathrm{He}$. The temperature program was $120^{\circ} \mathrm{C}$ initially, holding $0.5 \mathrm{~min}$, ramping at the rate of $10^{\circ} \mathrm{C} / \mathrm{min}$ until $310^{\circ} \mathrm{C}$, and holding for $2 \mathrm{~min}$. Masses were analysed over the range 40-350 m/z.

Viscosity at different temperature was measured using The Brookfield DV-III Rheometer and following the ASTM D445 standard test method. Brookfield Rheometer was controlled externally via accompanying software on a computer. The working principle of the DV-III Rheometer is to drive a spindle (which is immersed in the test fluid) through a calibrated spring. The viscous drag of the fluid against the spindle is measured by the spring deflection is measured using rotary transducer. The temperature of the sample is controlled by an oil bath connected with a sample cup. Experiments were carried out from 20 to 100 ${ }^{\circ} \mathrm{C}$ at $5{ }^{\circ} \mathrm{C}$ intervals. In order to reduce the experimental error all experiments were replicated for 3 times and the average data were taken.

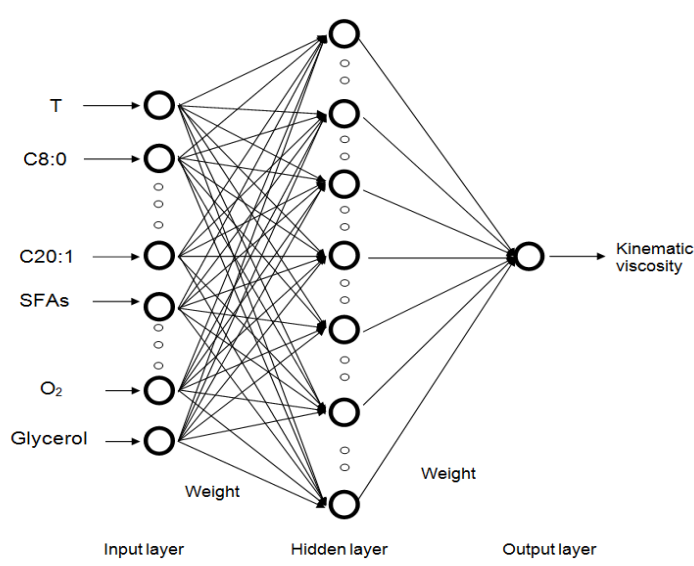

Figure 2: ANN model for kinematic viscosity prediction of biodiese 
ANN represents a mathematical relationship between input and output parameters of a system as like a black box model. The selection of input parameters which contribute to the output is therefore a crucial task. It is also desirable to minimize the number of input parameters for an ANN system in order to reduce the computational time. In general, the best input parameters are being selected based on the understanding of the physics of a problem. Published literatures, experimental works and numerical modeling have prove that kinematic viscosity of biodiesel is a function of temperature, chemical composition and fatty acid profile of methyl ester. In the present study, 23 variables are used as input parameters in developing of ANN for viscosity prediction. These parameters are include: temperature (T), saturation factor (SFA), monounsaturation factor (MUFA), poli-unsaturatin factor (PUFA), unsaturation factor (UFS), iodine value (IV), saponification value (SV), average chain length (ACL), carbon $(\mathrm{C})$, hydrogen $(\mathrm{H})$, oxygen $(\mathrm{O})$, glycerol content and mass percentage of 11 fatty acid methyl esters that are commonly found in the biodiesel.

The feed-forward learning algorithm back-propagation was applied for the single hidden layer shown in the Figure 2. The Neural Network has been optimized using the MATLAB Version R2012a Neural Network Toolbox. The Number of hidden neurons in the hidden layer is a crucial parameter. If the number is too small, the ANN may not reflect the complex relationship between input and output value. On the contrary, a large number may create such a complex network that might lead to a very large output error caused by over fitting of the training sample set. In the present study, the number neurons in the hidden layer have been optimized using trial and error methods during the training stage. Neurons in the input layer have no transfer function. A logistic sigmoid (logsig) transfer function has been used in hidden layer while purelinear (purelin) transfer function has been used in output layer.

Using the results produced by the network, statistical methods have been used to investigate the prediction performance of $\mathrm{NN}$ results. To judge the prediction performance of a network, several performance measures are used. Those include statistical analysis in terms of absolute fraction of variance $\left(R^{2}\right)$, root mean squared $(R M S)$ and maximum average error percentage $(M A E P)$. Formulas to calculate the error parameters are shown in Equation 2 to 4.

$$
\begin{aligned}
& R^{2}=1-\left(\frac{\sum_{i=1}^{i=N}\left(E_{a}-E_{p}\right)^{2}}{\sum_{I=1}^{I=N}\left(E_{a}-E_{M}\right)^{2}}\right) \\
& R M S=\sqrt{\frac{\sum_{i=1}^{i=N}\left(E_{a}-E_{p}\right)^{2}}{N}} \\
& \mathrm{MEP}=\frac{1}{\mathrm{~N}} \sum_{i=1}^{i=N}\left(\frac{\left|E_{a}-E_{p}\right|}{E_{a}} \times 100\right)
\end{aligned}
$$

Where, $E_{a}$-Actual result; $E_{p}$-Predicted result; $E_{m}$-Mean value; $N$-Number of pattern

\section{RESULTS AND DISCUSSION}

The ANN model establishes a relationship between input and output variables. The ranges of variation in the experimental data in ANN training processes are crucial for the robustness of the prediction ability. Table 1 shows the measured fatty acid profile of 10 biodiesel samples that have been used to train the ANN model for kinematic viscosity prediction. The fatty acid methyl esters ranges from 8 to 20 carbon chain length are quite evenly distributed in the samples. The average carbon chain lengths of biodiesels are ranges from 8.90 to 17.94 as shown in Table 1. The chemical compositions of the samples are also combined with saturated and unsaturated fatty acid methyl esters. The unsaturated fraction of fatty acid methyl esters (UFA) are range from 0 to 91.93 by weight percentage where mono-unsaturation factors (MUFA) and poli-unsaturation factors (PUFA) range from 0 to 73 and 0 to 55.04 respectively. The other chemical properties including iodine value (IV), soponification value (SV), average chain length (ACL), carbon (C), hydrogen (H), oxygen (O) and glycerol larger ranges as shown in Table 1. Therefore the results of chemical composition which illustrate that the 10 biodiesel samples are suitable for developing robust ANN prediction model for predicting temperature dependent biodiesel viscosity.

Figure 3 graphically shows the experimental results of the kinematic viscosity of ten biodiesel samples at different temperature ranging from $20^{\circ} \mathrm{C}$ to $100^{\circ} \mathrm{C}$. It can be seen from Figure 4 that the kinematic viscosity decreases for all biodiesel samples with the increase of temperature. The reduction of kinematic viscosity is prominent in the temperature range $20{ }^{\circ} \mathrm{C}$ to $50{ }^{\circ} \mathrm{C}$, while it has less influence on temperature in the range of $50{ }^{\circ} \mathrm{C}$ to $100{ }^{\circ} \mathrm{C}$. This can be explained by a change in chemical composition of biodiesel because of 
Jahirul et al., An Artificial Neutral Network (ANN) Model for Predicting Biodiesel Kinetic Viscosity as a Function of Temperature and Chemical Compositions

Table 1: Chemical properties of the 10 biodiesel samples

\begin{tabular}{lcccccccccc}
\hline & \multirow{2}{*}{ COME } & CSOME & SOME & \multicolumn{7}{c}{ SOMOME } \\
\cline { 5 - 10 } & & & & $\mathbf{1}$ & $\mathbf{2}$ & $\mathbf{3}$ & $\mathbf{4}$ & $\mathbf{5}$ & $\mathbf{6}$ & $\mathbf{7}$ \\
\hline C8:0 (wt\%) & 0 & 0 & 0 & 55.12 & 0.31 & 0 & 27.72 & 0.16 & 27.56 & 0 \\
C10:0 (wt\%) & 0 & 0 & 0 & 44.77 & 0.75 & 0 & 22.76 & 0.37 & 22.39 & 0 \\
C12:0 (wt\%) & 0.31 & 0 & 0 & 0 & 72.89 & 0 & 36.45 & 36.45 & 0 & 0 \\
C14:0 (wt\%) & 0.35 & 0.53 & 0 & 0 & 23.61 & 0 & 11.81 & 11.80 & 0 & 0.03 \\
C16:0 (wt\%) & 11.93 & 2.51 & 11.08 & 0 & 2.350 & 20.52 & 1.17 & 11.44 & 10.26 & 4.45 \\
C16:1(wt\%) & 0.22 & 0.55 & 0.18 & 0 & 0 & 0 & 0 & 0 & 0 & 0.12 \\
C18:0 (wt\%) & 2.43 & 4.13 & 5.23 & 0 & 0 & 9.00 & 0 & 4.50 & 4.50 & 2.53 \\
C18:1 (wt\%) & 55.72 & 35.78 & 33.82 & 0 & 0 & 66.08 & 0 & 33.04 & 33.04 & 72.09 \\
C18:2 (wt\%) & 26.87 & 55.04 & 49.38 & 0 & 0 & 4.20 & 0 & 2.10 & 2.10 & 19 \\
C20:0 (wt\%) & 0.320 & 0.31 & 0.18 & 0 & 0 & 0 & 0 & 0 & 0 & 0.49 \\
C20:1 (wt\%) & 0.570 & 0.30 & 0 & 0 & 0 & 0 & 0 & 0 & 0 & 1.03 \\
SFA (wt\%) & 15.55 & 7.68 & 16.62 & 99.89 & 99.91 & 29.52 & 99.90 & 64.72 & 64.71 & 7.69 \\
MUFA (wt\%) & 56.51 & 36.63 & 34.00 & 0.00 & 0.10 & 66.08 & 0.05 & 33.09 & 33.04 & 73.24 \\
PUFA (wt\%) & 26.87 & 55.04 & 49.38 & 0.00 & 0.00 & 4.20 & 0.00 & 2.10 & 2.10 & 18.69 \\
UFA (wt\%) & 83.38 & 91.67 & 83.38 & 0.00 & 0.10 & 70.28 & 0.05 & 35.19 & 35.14 & 91.93 \\
IV & 99.47 & 132.67 & 120.04 & 0.00 & 0.11 & 67.03 & 0.06 & 33.57 & 33.52 & 99.65 \\
SV & 199.15 & 198.43 & 201.14 & 359.61 & 270.70 & 201.93 & 315.15 & 236.32 & 280.77 & 198.35 \\
ACL & 17.56 & 17.83 & 17.78 & 8.90 & 12.54 & 17.59 & 10.72 & 15.06 & 13.24 & 17.94 \\
C (wt\%) & 75.65 & 76.27 & 76.61 & 67.94 & 72.40 & 76.04 & 70.17 & 74.22 & 71.99 & 76.28 \\
H (wt\%) & 11.91 & 11.75 & 11.90 & 11.40 & 12.15 & 12.23 & 11.78 & 12.19 & 11.82 & 12.01 \\
O (wt\%) & 11.38 & 11.33 & 11.49 & 20.54 & 15.46 & 11.53 & 18.00 & 13.50 & 16.04 & 11.33 \\
Glycerol & 1.03 & 0.58 & 0 & 0 & 0 & 0 & 0 & 0 & 0 & 0.38 \\
\hline
\end{tabular}

crystallization of high melting compounds at low temperature. Figure 3 also shows that the kinematic viscosities of biodiesel samples are significantly different from one another. This is mainly due to the different in chemical composition. The canola biodiesel showed the highest kinematic viscosities followed by tallow, FPOME-7, cottonseed oil, FPOME-3, soybean, FPOME-5, FPOME-2, FPOME-6, FPOME-4 and FPOME-1. It is well known that total glycerol content increases kinematic viscosity of biodiesel which has been evident in the experimental results found in this study. Similarly, other chemical compositions also have a similar effect experimental result. It is interesting to note that the kinematic viscosity at $40{ }^{\circ} \mathrm{C}$ for all biodiesels is within the European $(3.0-5.0 \mathrm{cSt})$ or ASTM $(1.9-6.0 \mathrm{cSt})$ standard limit except canola biodiesel. Wide ranges of kinematic viscosity shown in figure 3 indicate the suitability of the experimental data for developing a robust ANN prediction model.

Using the experimental data obtained in this study, the structure of the developed ANN model is shown in Figure 4. While developing the ANN model, 204 data sets obtained from experimental work in this study have been used. Among those, $70 \%$ of the data sets were used randomly to train the network, $15 \%$ of data sets were used validation and $15 \%$ of data were used as training data sets. Twenty three neurons representing input

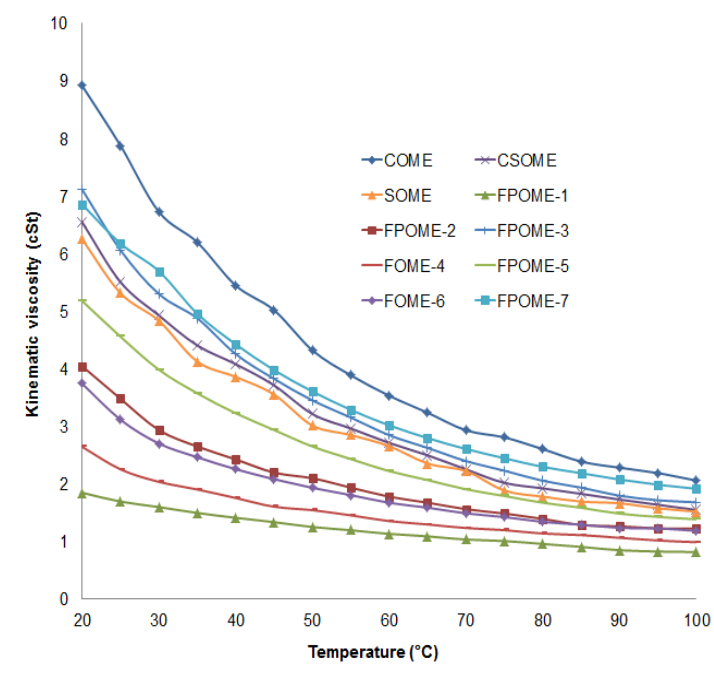

Figure 3: Kinematic viscosity of biodiesel samples at different temperature

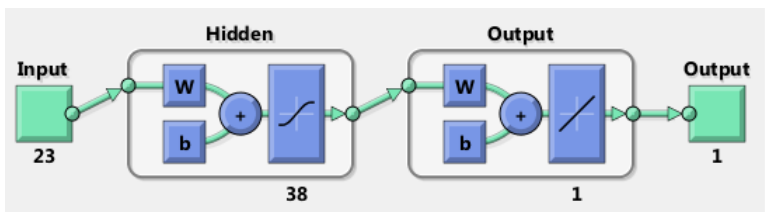

Figure 4: Optimised ANN for kinematic viscosity prediction 
parameters including temperature and chemical properties shown in Table 1 where used as input parameters and corresponding kinematic viscosity shown in Figure 3 where used in single output neuron. The prediction accuracy of the model was observed for 23 to 46 neurons in the single hidden layer. It was found the prediction accuracy improved with the number neurons in the hidden layer. The best result was found for 38 neurons in the hidden layer. The computational time increased significantly along with the over fitting problem which eventually reduces the models effectiveness. The training was stopped after 27 iterations as shown in Figure 5 to avoid over fitting of the network. After stopping the training the software has identified the best ANN network at 21 iterations.

The ability of the ANN model to predict kinematic viscosity has been verified by simulating it with unknown input variables. For this purpose experiments were conducted on a new biodiesel derived from waste cooking oil (WCOME) which was not used during the training of ANN model. The predicted kinematic viscosity of WCOME at temperatures from 20 to $100{ }^{\circ} \mathrm{C}$ were compared with the corresponding experimental values and shown in Figure 6. Actual and predicted data shown in Figure 6 indicates the good prediction ability of the developed ANN for predicting kinematic viscosity of biodiesel at different temperatures. In statistical analysis it was found that the absolute fraction of variance $\left(R^{2}\right)$ was close to unity 0.978 , Root-Mean-Squared $(R M S)$ error was $0.257 \mathrm{cSt}$ and maximum average error percentage $(M A E P)$ was $5.27 \%$. This is a good correlation between the measured and predicted kinematic viscosity. The network has been trained very well and can be used to simulate biodiesel kinematic viscosity over a wide range of temperatures. However, the prediction accuracy of the model should be further improved by increasing the number and ranges of training data set.

\section{CONCLUSION}

The aim of this paper is to investigate the ability of using artificial neural networks (ANN) for prediction of biodiesel kinetic viscosity from its chemical compositions. A standard back propagation (BP) neural network model with LM algorithms was developed. To train the network, biodiesels from ten different feedstocks have been characterized by using standard experimental methods and equipments. Important chemical properties and temperature were used as input variables to output the corresponding kinetic viscosity. The performance of the developed ANN prediction model was evaluated by prediction with the experimental results which were not used in the training process. The network produces the predicted results in good argument to the experimental ones. The overall results show that the networks can be used as an alternative way for predicting kinematic viscosity of biodiesel at different temperature conditions. The absolute fraction of variance $\left(R^{2}\right)$, RootMean-Squared (RMS) and maximum average error percentage $(M A E P)$ was values were found $0.978,0.257 \mathrm{cSt}$ and $5.27 \%$ respectively which is within an acceptable range of accuracy. The results of this study also show that ANN has ability to learn and generalize a wide range of experimental conditions. Therefore, the usage of ANNs may be recommended to optimize the chemical composition of biodiesels to optimize fuel quality for internal combustion engine application. However the network should be further improved by including additional robust data set during the training process.

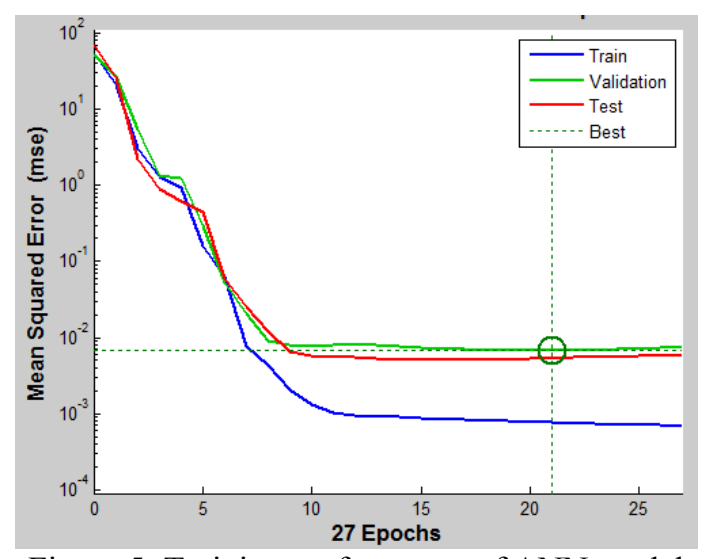

Figure 5: Training performance of ANN model

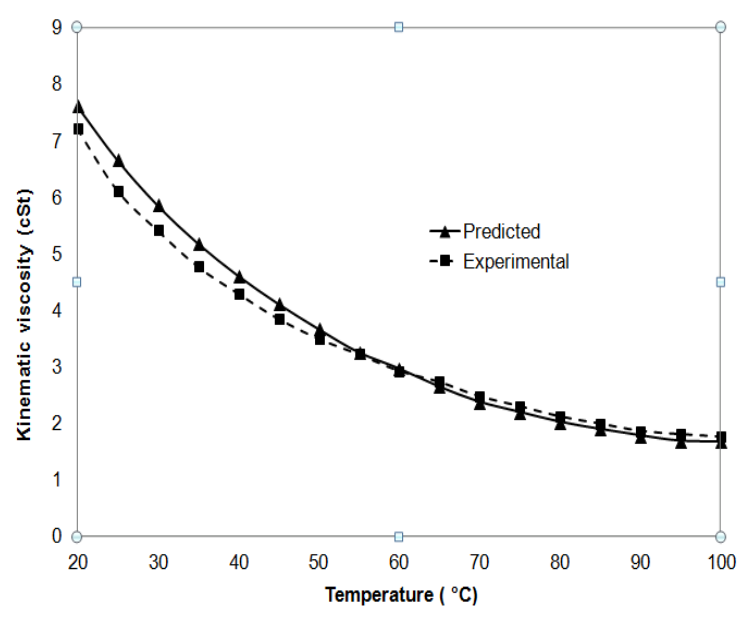

Figure 6: Experimental and predicted kinematic viscosity of WCOME 


\section{ACKNOWLEDGMENTS}

The authors wish to express their thanks to QUTPRA scholarship, Faculty of Science and Engineering (SEF), Queensland University of Technology (QUT) and Australian Research Council for providing fund for conducting this study. The authors would also like to thank Mr Scott Abbert his assistance during experimental work.

\section{REFERENCES}

Alkim, E.; Gürbüz, E.; Kılıç, E. (2012) A fast and adaptive automated disease diagnosis method with an innovative neural network model. Neural Netw. 330, 88-96.

Arcakhoglu, E., Cavusoglu, A., Erisen, A., (2004) Thermodynamic analyses of refrigerant mixtures using artificial neural-networks. Appl Energ, 2004. vol. 78, pp. 219-30.

Basheer, I. A., Hajmeer, M (2000) Artificial neural networks: fundamentals, computing, design, and application. Journal of Microbiological Methods 43(1), 3-31

Carrillo, S.; Harkin, J.; McDaid, L.; Pande, S.; Cawley, S.; McGinley, B.; Morgan, F. (2012) Advancing interconnect density for spiking neural network hardware implementations using traffic-aware adaptive network-on-chip routers. Neural Netw. 330, 42-57.

Costa, M.A.; Braga, A.P.; Menezes, B.R. (2012) Convergence analysis of sliding mode trajectories in multiobjective neural networks learning. Neural Netw. 330, 21-31.

Christo, F.C.; Masri, A.R.; Nebot, E.M. (1996) Artificial neural network implementation of chemistry with pdf simulation of H2/CO2 flames, Combustion and Flame, 106(4), 406-427.

Demirbas, A., (2008). Relationships derived from physical properties of vegetable oil and biodiesel fuels. Fuel, 87, 1743-1748

Fernando S, Karra P, Hernande R., Kumar J. S., (2007) Effect of incompletely converted soybean oil on biodiesel quality, Energy 32, 844-851.

Haseeb, A. S. M. A., Fazal, M. A., Jahirul, M. I., \& Masjuki, H. H. (2011). Compatibility of automotive materials in biodiesel: A review. Fuel, 90(3), 922-931

Hoekman, S. K., Broch, A., Robbins, C., Ceniceros, E., \& Natarajan, M. (2012). Review of biodiesel composition, properties, and specifications. Renewable and Sustainable Energy Reviews, 16(1), 143169.

Jahirul, M.I.; Brown, R.J.; Senadeera, W.; O'Hara, I.M.; Ristovski, Z.D. (2013) The Use of Artificial Neural Networks for Identifying Sustainable Biodiesel Feedstocks.Energies, 6, 3764-3806.

Knothe, G.; Steidley, K. R. (2007) Kinematic viscosity of biodiesel components fatty acid alkyl esters and related compounds at low temperatures. Fuel, 86(16), 2560-2567.

Knothe, G.; Steidle, K. R. (2005) Kinematic viscosity of biodiesel fuel components and related compounds Influence of compound structure and comparison to petrodiesel fuel components. Fuel, 84(9), 10591065.

Kreider J.F, Wang XA.. Artificial neural networks demonstrations for automated generation of energy use predictors for commercial buildings. ASHRAE Transactions. 1992. vol. 97(1), pp. 775-9.

Lee, S.; Tanaka, D.; Kusaka, J.; Daisho, Y. (2002) Effects of diesel fuel characteristics on spray and combustion in a diesel engine. JSAE, 23, 407-414.

Minnett, R.C.J.; Smith, A.T.; Lennon, W.C., Jr.; Hecht-Nielsen, R. (2011) Neural network tomography: Network replication from output surface geometry. Neural Netw. 245, 484-492.

Refaat, A.A. (2009) Correlation between the chemical structure of biodiesel and its physical properties. Int $J$ Environ Sci Technol, 6, 677-694. 